\title{
Use of a case-control study and control bank to investigate an outbreak of locally acquired cyclosporiasis in Canada, 2016
}

\author{
V Morton ${ }^{1 *}$, K Meghnath², M Gheorghe², A Fitzgerald-Husek ${ }^{3}$, J Hobbs ${ }^{4}$, L Honish ${ }^{5}$, S David ${ }^{6}$
}

\begin{abstract}
Background: Cyclospora is an intestinal parasite that is not endemic in Canada. However, national outbreaks of locally acquired cases have been reported since 2013. These outbreaks were suspected to be associated with consumption of produce imported from countries where Cyclospora is endemic. Identification of the source can be challenging because of reporting delays and limited traceability of produce.
\end{abstract}

Objective: To report on a national outbreak of locally acquired cyclosporiasis, highlight the challenges of investigating these outbreaks and document the first time use of a control bank to recruit controls for a national outbreak case-control study in Canada.

Methods: Cases of cyclosporiasis were identified through provincial laboratory testing and reported through provinces to the national level. Cases were interviewed about food exposures using a questionnaire and food exposures reported by cases were compared to Foodbook reference values. To narrow down the food items of interest, a matched case-control study was conducted. Controls for the study were recruited primarily from a control bank, that is, a list of individuals who had previously agreed to participate in public health-related surveys.

Results: In total, 87 cases of locally acquired cyclosporiasis with onset or report dates between May 19, 2016 and August 10, 2016 were reported by four provinces. Comparing case exposures to Foodbook reference values identified several food items of interest, including blackberries, other berries, herbs and leafy greens. The case-control study identified only blackberries and mesclun greens as significantly more frequently consumed by cases than controls. Due to lack of product details for blackberries and mesclun greens, the source of the outbreak was not conclusively identified.

Conclusion: Blackberries were the primary food item of interest, but could not be identified as the conclusive source due to lack of traceability. The control bank was found to be a useful tool for control recruitment.
This work is licensed under a Creative Commons Attribution 4.0 International License.

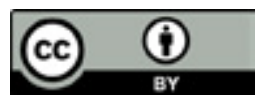

Affiliations

1 Public Health Agency of Canada, Guelph, ON

2 Public Health Agency of Canada, Ottawa, ON

${ }^{3}$ York Region Public Health, Newmarket, ON

${ }^{4}$ Public Health Ontario, Toronto, ON

${ }^{5}$ Alberta Health Services,

Edmonton, $A B$

${ }^{6} \mathrm{BC}$ Centre for Disease Control, Vancouver, BC

\section{${ }^{\star}$ Correspondence:}

vanessa.morton@canada.ca

Suggested citation: Morton V, Meghnath K, Gheorghe M, Fitzgerald-Husek A, Hobbs J, Honish L, David S. Use of a case-control study and control bank to investigate an outbreak of locally acquired cyclosporiasis in Canada, 2016. Can Commun Dis Rep 2019;45(9):225-9. https://doi.org/10.14745/ccdr.v45i09a01

Keywords: Cyclospora, outbreak, case-control study, cyclosporiasis, Canada

\section{Introduction}

Cyclospora cayetanensis is an enteric parasite that causes gastroenteritis (1). Cyclospora is not endemic in Canada; cases are often associated with international travel. Locally acquired cases of cyclosporiasis are likely associated with consumption of produce imported from countries where Cyclospora is endemic (2). For instance, in the 1990s there were several outbreaks associated with raspberries imported from Guatemala; import restrictions put an end to these outbreaks (3). Since 2013, national outbreaks of locally acquired cyclosporiasis have occur in Canada each spring/summer. However, identification of the 
source has been difficult because of lack of molecular typing methods, considerable case reporting delays and limited traceability of produce.

An important step in foodborne outbreak investigation is hypothesis generation, and one hypothesis generation technique is to compare food exposures reported by cases to exposures reported by the general population (4). In Canada, investigators can use Foodbook reference values for comparisons. Foodbook is a population-based telephone survey that was conducted in all Canadian provinces and territories over a one-year period (2014-2015) with a primary focus of describing what foods Canadians eat over a seven-day period. Case-control studies are also commonly used for hypothesis generation during outbreak investigations. However, they can be lengthy and costly to conduct. One of the challenges associated with these studies is having an effective method to recruit controls. A control bank was created as part of the Foodbook study in order to address this challenge. Participants were asked if they were willing to be contacted for future outbreak investigation or research studies. The use of a control bank to recruit study participants was successfully used in Australia for outbreak investigations and other public health research projects in Australia $(5,6)$.

The objective of this report is to describe the investigation into a national outbreak of locally acquired cyclosporiasis in 2016 and highlight some of the challenges associated with cyclosporiasis outbreak investigation in Canada.

\section{Outbreak detection}

On June 27, 2016, public health officials in British Columbia notified the Public Health Agency of Canada (PHAC) of their first reported case of locally acquired cyclosporiasis that year. By July 9, 2016, Ontario and Alberta had reported eight locally acquired cases. PHAC, in collaboration with local, provincial and federal partners, promptly launched a national outbreak investigation.

\section{Methods}

\section{Epidemiological investigation}

An outbreak-associated case of cyclosporiasis was defined as laboratory confirmation of infection with $C$. cayetanensis in a resident of or visitor to Canada, with symptom onset on or after April 1, 2016, and no history of travel outside of Canada or the United States during the 14 days preceding symptom onset. Cases were identified per provincial diagnosing standards and reported to PHAC by provincial public health officials. Data on food exposures in the 14 days preceding illness onset were obtained using a cyclosporiasis-specific questionnaire. Certain cases were re-interviewed by centralized interviewers at the national or local level if the initial interviews did not obtain the required details on exposures or product. Data were collected on exposure to fresh berries, herbs, leafy greens, peas and other vegetables; only exposure to fresh produce was considered. Exposure frequencies were compared to food reference values from the Foodbook study for the months of May, June, July and August for the affected provinces using binomial probabilities and significance thresholds of $p=0.05(7,8)$.

\section{Case-control study}

A case-control study was conducted to identify food items associated with illness. Cases were eligible for inclusion in the study if they met the outbreak case definition and had both a reasonably complete questionnaire and a known date of illness onset. Cases also had to have an onset date after May 31, 2016, and the illness had to have been reported on or before September 26, 2016. Controls were matched to each case based on age group (10-19, 20-69 and $\geq 70$ years) and location using the first digit of the postal code (9). Controls were asked about food exposures during the same 14-day period as cases. Three controls were selected for each case in order to maximize the statistical power of the results based on the estimated sample size. Controls were recruited using a control bank, a repository of contact information for people who were interviewed for the Foodbook study and who had consented to be contacted for future research or investigative purposes. Additional recruitment, once the control bank was exhausted, was conducted via random digit dialling.

Case-control data were analyzed using McNemar's odds ratios (ORs) for matched pairs. Factors identified in univariable analysis with ORs greater than one and a p-value of less than 0.2 were included in a multivariable regression model. Factors were removed using backward stepwise selection; variables remained in the model if they changed the significant coefficients by more than $20 \%(10,11)$.

\section{Results}

\section{Epidemiological investigation}

In total, 87 cases of locally acquired cyclosporiasis were identified in four provinces (Ontario, $n=75$; Quebec, $n=8$; Alberta, $n=2$; British Columbia, $n=2)$. Onset or specimen collection dates ranged from May 19 to August 10, 2016 (Figure 1). One case was excluded from the food history because of the uncertainty around the onset date (reported as "mid-July") and the long delay between the exposure period and interview (isolation date was September 23, 2016).

The median age of cases was 51 years (range $=15-89$ years) and $52 \%$ were female. One case was hospitalized. There were no deaths. 
Figure 1: Number of confirmed outbreak cases of locally acquired cyclosporiasis by symptom onset or specimen collection date and status of inclusion/exclusion in the case-control study, 2016, Canada

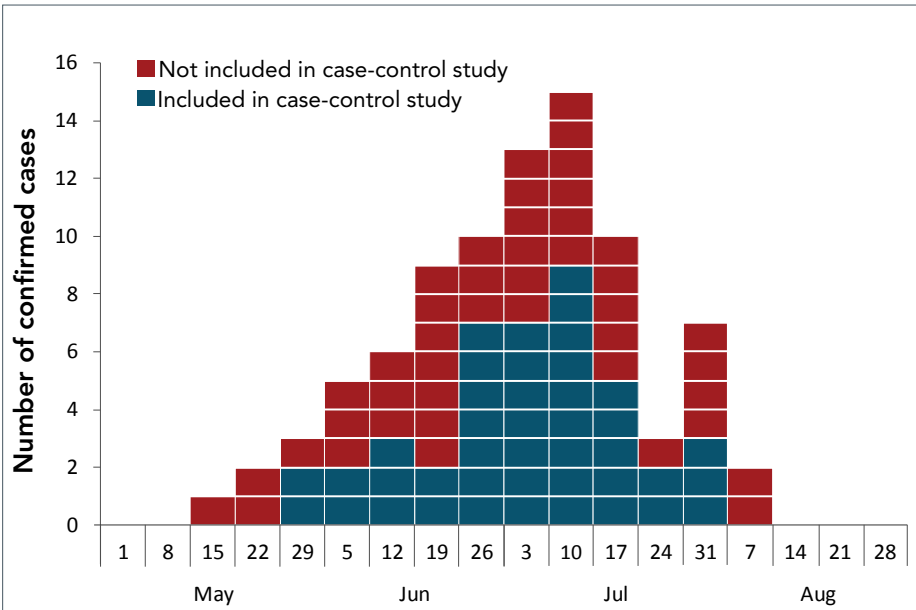

Week of symptom onset or specimen collection $(n=86)$

a One outbreak case was excluded as no symptom onset date or specimen collection date was available; only an isolation date was available

Table 1: Results of binomial probability comparison between Foodbook reference values and odds ratio results from case-control study

\begin{tabular}{|c|c|c|c|c|c|c|c|}
\hline \multirow[b]{2}{*}{ Food item } & \multicolumn{3}{|c|}{ Foodbook reference (seven-day recall)a } & \multicolumn{4}{|c|}{ Case-control study (14-day recall) ${ }^{b}$} \\
\hline & $\begin{array}{c}\text { Cases } \\
\text { exposed (\%) }\end{array}$ & $\begin{array}{c}\text { Controls }{ }^{c} \\
\text { exposed }(\%)\end{array}$ & $\begin{array}{c}\text { Binomial } \\
\text { probability } \\
\text { (p-value) }\end{array}$ & $\begin{array}{c}\text { Cases exposed } \\
(\%)\end{array}$ & $\begin{array}{c}\text { Controls } \\
\text { exposed (\%) }\end{array}$ & Odds ratio & $\mathrm{p}$-value \\
\hline Strawberries & 69 & 64 & 0.07 & 68 & 79 & 0.57 & 0.16 \\
\hline Raspberries & 51 & 30 & $<0.001$ & 53 & 50 & 1.09 & 0.81 \\
\hline Blackberries & 43 & 11 & $<0.001$ & 49 & 28 & 2.50 & 0.02 \\
\hline Other berries & 69 & No datad & NA & 73 & 59 & 1.86 & 0.12 \\
\hline Basil & 23 & 22 & 0.11 & 24 & 35 & 0.60 & 0.23 \\
\hline Cilantro & 42 & 19 & $<0.001$ & 38 & 39 & 0.96 & 0.93 \\
\hline Parsley & 47 & 33 & 0.01 & 53 & 39 & 1.78 & 0.16 \\
\hline $\begin{array}{l}\text { Other fresh } \\
\text { herbs }\end{array}$ & 27 & No datad & NA & 32 & 24 & 1.48 & 0.37 \\
\hline Iceberg lettuce & 39 & 44 & 0.06 & 44 & 64 & 0.45 & 0.03 \\
\hline Romaine lettuce & 73 & 53 & $<0.001$ & 76 & 84 & 0.60 & 0.27 \\
\hline Spinach & 48 & 33 & 0.003 & 50 & 67 & 0.49 & 0.06 \\
\hline Mesclun greens & 30 & 18 & 0.003 & 30 & 15 & 2.50 & 0.03 \\
\hline Arugula & 42 & No datad & NA & 46 & 31 & 1.92 & 0.13 \\
\hline $\begin{array}{l}\text { Other lettuce or } \\
\text { leafy greens }\end{array}$ & 43 & No datad & NA & 46 & 42 & 1.18 & 0.66 \\
\hline Peas & 15 & 29 & 0.002 & 20 & 37 & 0.42 & 0.05 \\
\hline Snow peas & 10 & No data ${ }^{d}$ & NA & 11 & 26 & 0.35 & 0.17 \\
\hline Snap peas & 17 & No data ${ }^{d}$ & NA & 21 & 20 & 1.04 & 0.94 \\
\hline
\end{tabular}

Exposure data were available for 86 cases. The most frequently strawberries (51/74, 69\%) and other berries $(51 / 74,69 \%)$ (Table 1). Compared with Foodbook reference values, cases with cyclosporiasis reported the following seven food exposures significantly $(p \geq 0.05)$ more frequently than did the general lettuce, spinach and mesclun greens.

\section{Case-control study}

Forty-two cases met the inclusion criteria for the case-control study. They were matched with 126 controls (117 from the control bank and nine enrolled via random digit dialling). The response rates for the control bank and random digit dialling were $60 \%$ and $24 \%$, respectively.

Univariate matched analysis indicated that cases were significantly more likely than controls to have been exposed to blackberries $(\mathrm{OR}=2.50 ; 95 \%$ confidence intervals $[\mathrm{Cl}]=$ $1.16-4.91)$ and mesclun greens $(\mathrm{OR}=2.50 ; 95 \% \mathrm{Cl}=1.01-5.48)$ (Table 1). Multivariate analysis using backward stepwise selection resulted in only exposure to blackberries being significant, with exposure to mesclun greens and arugula included in the model as statistical confounders. reported food items were romaine lettuce $(51 / 70,73 \%)$, population: raspberries, blackberries, cilantro, parsley, romaine 


\section{Food safety investigation}

Based on the results of the case-control study, attempts were made to trace food items that were significantly more likely to have been consumed by cases. Brand information, purchase dates, loyalty card data and store records were used. Import data were requested for blackberries and mesclun greens for May through August 2016; import data for mesclun greens were not available.

A common country of origin or supplier of blackberries was not identified based on the limited information available. Mexico was the largest supplier of blackberries in May, accounting for $64 \%$ of Canadian imports. This market share decreased to $20 \%$ in June and 3\%-4\% in July and August; during July and August the majority of blackberries imported into Canada were imported from the United States. Guatemala accounted for $0.2 \%$ of blackberry imports from May to August.

\section{Public health response}

The public was notified of the outbreak of locally acquired cyclosporiasis through a Public Health Notice posted on the PHAC website. As no specific product was identified, general prevention recommendations were provided. These recommendations encourage cooking produce imported from countries where Cyclospora is common and consuming fresh produce grown in countries where Cyclospora is not common. People travelling to a country where Cyclospora is found were advised to avoid food that has been washed in local drinking water, to drink water from a safe source and to eat cooked food or fruit that can be peeled.

\section{Discussion}

This outbreak investigation demonstrates some of the challenges associated with identifying the source of cyclosporiasis outbreaks. Despite a thorough investigation, the source of this outbreak was not identified. However, both the Foodbook exposure information and the case-control study were useful in generating hypotheses. To our knowledge, this outbreak was the first time a case-control study was conducted as part of an investigation into a national cyclosporiasis outbreak in Canada, and the control bank proved to be an effective tool for recruiting controls.

Although blackberries were not conclusively identified as the source of the outbreak, they were identified as a food item of interest via both the Foodbook reference and the case-control study. In addition, the case-control study helped to strengthen the evidence for blackberries as a suspect source. The reason these findings alone could not prove that blackberries were the source of the outbreak was the lack of specificity on the brand or producer of blackberries consumed by cases. These findings were consistent with previous locally acquired cyclosporiasis outbreaks in Canada in 2014 and 2015 where blackberries were identified as one of multiple suspected sources (Public Health Agency of Canada. Multi-provincial outbreak investigation of Cyclosporiasis in British Columbia, Quebec and Ontario: Final Epidemiological Summary, unpublished report). This finding is also supported by examination of blackberry import data, which revealed that Mexico was a large supplier of blackberries to Canada in May and early June. When taking into account the incubation period of cyclosporiasis (up to 14 days) and the shelf life of blackberries (up to 21 days), illnesses associated with these blackberries could have occurred more than a month after importation, lining up with the peak of the outbreak in July $(3,12)$. Cyclospora is endemic in Mexico, and cyclosporiasis cases have been reported among Canadians travelling to Mexico (13). It is probable that one or more additional food items also contributed to this outbreak as only $43 \%$ of cases reported blackberry consumption and the percentage of cases who reported consuming blackberries decreased among those who reported later onset dates.

Both the Foodbook comparison and the case-control analysis identified mesclun greens as another item of interest. However, interpretation of this finding was limited by the lack of standardization in the types of greens identified as "mesclun greens" and a lack of packaging or brand details; this precluded positive identification of a specific product.

\section{Strengths and limitations}

This outbreak demonstrated the usefulness of a control bank for recruitment of controls for a case-control study. Having a list of persons willing to participate in surveys facilitated rapid and cost-effective initiation of a case-control study. In addition, the response rate for the control bank was much higher than random digit dialling; the decrease in household landline use and the increased availability of call display has made it more difficult to recruit individuals using random digit dialling. However, the use of a control bank introduces the possibility of selection bias as individuals who agree to participate in the control bank may differ from the general population.

One other important limitation to note is that the time periods for the food history for the case-control study data and the Foodbook data differed: Foodbook participants were asked about a seven-day food history, whereas cases in this investigation were asked about exposures over 14 days based on the Cyclospora incubation period. This difference in recall period could have resulted in more food items being reported at higher frequencies by the cases than the reference population from Foodbook.

\section{Next steps}

Case-control studies are a helpful tool in investigation as they can identify a food source that was more likely to have been eaten by cases than by controls; however, additional laboratory and/or traceback information is required to definitively identify the source. 
This investigation also highlights the need to revisit strategies for effective investigation of locally acquired cyclosporiasis. Collecting good food exposure data and purchase information from cases at the time of initial case interviewing is important but challenging given the long incubation period and limited shelf life of produce. The lack of routinely available laboratory methods to type Cyclospora is another major investigative challenge. One possible solution would be to focus efforts on identifying and investigating event- or premise-based clusters where cases are more likely to have contracted their illness from one contaminated food product. Further discussion with produce-exporting countries where Cyclospora is endemic might help to address prevention and control measures.

\section{Conclusion}

Results from this outbreak investigation have contributed to the understanding of this disease in Canada and demonstrated that a control bank can be an effective tool for conducting case-control studies as part of public health investigations.

\section{Authors' statement}

VM - Investigation, methodology, writing (original draft and review \& editing)

KM and MG - Investigation, methodology, writing (review \& editing)

$\mathrm{AFH}, \mathrm{JH}, \mathrm{LH}$ and SD — Investigation, writing (review \& editing)

\section{Conflict of interest}

None.

\section{Acknowledgements}

We wish to thank colleagues from British Columbia Centre for Disease Control, Alberta Health, Alberta Health Services, Public Health Ontario, Ontario Ministry of Health and Long-Term Care, Ministère de la santé et des services sociaux du Québec, Canadian Food Inspection Agency, Health Canada and the Public Health Agency of Canada as well as public health laboratories in British Columbia, Alberta, Ontario and Quebec.

\section{Funding}

This work was supported by the Public Health Agency of Canada.

\section{References}

1. Heymann $\mathrm{DL}$, editor. Control of communicable diseases manual. 20th edition. Washington (DC): American Public Health Association; 2014.

2. Hedberg CW, Osterholm MT. Foodborne outbreaks caused by Cyclospora: the message is more important than the messenger. Epidemiol Infect 2016 Jul;144(9):1803-6. DOI PubMed

3. Herwaldt BL, Beach MJ; Cyclospora Working Group. The return of Cyclospora in 1997: another outbreak of cyclosporiasis in North America associated with imported raspberries. Ann Intern Med 1999 Feb;130(3):210-20. DOI PubMed

4. CIFOR. Guidelines for foodborne disease outbreak response (CIFOR Guidelines) [Internet]. Atlanta (GA): Council to Improve Foodborne Outbreak Response; 2009. https://cifor.us/products/guidelines

5. Munnoch SA, Ward K, Sheridan S, Fitzsimmons GJ, Shadbolt CT, Piispanen JP, Wang Q, Ward TJ, Worgan TL, Oxenford C, Musto JA, McAnulty J, Durrheim DN. A multi-state outbreak of Salmonella Saintpaul in Australia associated with cantaloupe consumption. Epidemiol Infect 2009 Mar;137(3):367-74. DOI PubMed

6. Stafford RJ, Schluter P, Kirk M, Wilson A, Unicomb L, Ashbolt R, Gregory $\mathrm{J}$ and the OzFoodNet Working Group. A multi-centre prospective case-control study of campylobac-ter infection in persons aged 5 years and older in Australia. Epidemiol Infect 2007 Aug;135(6):978-88. DOl PubMed

7. Centre for Food-borne, Environmental and Zoonotic Infectious Diseases, Infectious Disease Prevention and Control Branch. Foodbook report. Guelph (ON): Public Health Agency of Canada; 2015. http://publications.gc.ca/collections/collection_2016/ aspc-phac/HP40-146-2015-eng.pdf

8. Gaulin C, Levac E, Ramsay D, Dion R, Ismaïl J, Gingras S, Lacroix C. Escherichia coli O157:H7 outbreak linked to raw milk cheese in Quebec, Canada: use of exact probability calculation and casecase study approaches to foodborne outbreak investigation. J Food Prot 2012 May;75(5):812-8. DOI PubMed

9. Postal code: Detailed definition. Ottawa (ON): Statistics Canada; (Accessed 2018-09-17). http://www.statcan.gc.ca/pub/92195-x/2011001/other-autre/pc-cp/pc-cp-eng.htm

10. Rimhanen-Finne R, Niskanen T, Hallanvuo S, Makary P, Haukka K, Pajunen S, Siitonen A, Ristolainen R, Pöyry H, Ollgren J, Kuusi M. Yersinia pseudotuberculosis causing a large outbreak associated with carrots in Finland, 2006. Epidemiol Infect 2009 Mar;137(3):342-7. DOI PubMed

11. Dohoo I, Martin W, Stryhn H, editors. Methods in epidemiologic research. Charlottetown (PEI): VER Inc.; 2012.

12. Perkins-Veazie P, Collins JK, Clark JR. Shelf-life and quality of 'Navaho' and 'Shawnee' blackberry fruit stored under retail storage conditions. J Food Qual 1999;22(5):535-44. DOI

13. Nichols GL, Freedman J, Pollock KG, Rumble C, Chalmers RM, Chiodini P, Hawkins G, Alexander CL, Godbole G, Williams C, Kirkbride HA, Hamel M, Hawker JI. Cyclospora infection linked to travel to Mexico, June to September 2015. Euro Surveill 2015;20(43): DOI PubMed 